Mathematical Modelling and Analysis

Volume 6 Number 2, 2001, pages 310-320

(C) 2001 Technika

\title{
STABILITY OF PIECEWISE POLYNOMIAL COLLOCATION FOR VOLTERRA INTEGRO-DIFFERENTIAL EQUATIONS ${ }^{1}$
}

\author{
P. OJA, M. TARANG \\ Department of Applied Mathematics, University of Tartu \\ Liivi 2, Tartu 50409, Estonia \\ E-mail: peeter.oja@ut.ee, mare.tarang@ut.ee
}

Received September 27, 2001; revised October 30, 2001

\begin{abstract}
Numerical stability of the spline collocation method by piecewise polynomials for Volterra integro-differential equations is investigated. Stability conditions depending on collocation parameters and also on parameters of certain test equation are obtained. Results of several numerical tests are presented supporting theoretical results.
\end{abstract}

\section{INTRODUCTION}

Discretization methods in practical solving of differential and integral equations are applicable only if they are stable. The first results about stability of the collocation method by polynomial splines for Volterra integral equations are given in [4] and the most adequate ones seem to be in [5], see also references therein. Special case of smooth splines is treated in [6]. Such problem for Volterra integro-differential equations is considered in [3], but there is no case of piecewise polynomials, i.e. splines with possible discontinuities in knots. In this paper we will analyze the numerical stability of the piecewise polynomial spline collocation method in the case in which the mesh is uniform. Stability means here the boundedness of approximate solutions in uniform norm when the number of knots increases. It turns out that, while for Volterra integral equations the stability depends only on the collocation parameters, for Volterra integro-differential equations there is also dependence on the parameters of certain test equation. We present also the results of

\footnotetext{
${ }^{1}$ Research supported by the Estonian Science Foundation Grant No. 3926.
} 
several numerical tests where the method is applied to a widely used test equation. They support completely the theoretical results.

\section{THE SPLINE COLLOCATION METHOD}

Consider the Volterra integro-differential equation

$$
y^{\prime}(t)=f(t, y(t))+\int_{0}^{t} \mathcal{K}(t, s, y(s)) d s, \quad t \in[0, T],
$$

with the initial condition $y(0)=y_{0}$. The functions $f:[0, T] \times \mathbb{R} \rightarrow \mathbb{R}$ and $\mathcal{K}: S \times \mathbb{R} \rightarrow \mathbb{R}$ (where $S=\{(t, s): 0 \leq s \leq t \leq T\}$ ) with number $y_{0}$ are supposed to be given.

A mesh $0=t_{0}<t_{1}<\ldots<t_{N}=T$ will be used, with the choice of knots $t_{n}$ being dependent on $N$, since we want investigate the process $N \rightarrow \infty$. Denote $h_{n}=t_{n}-t_{n-1}$ and $\sigma_{n}=\left(t_{n-1}, t_{n}\right], n=1, \ldots, N, \Delta_{N}=\left\{t_{1}, \ldots, t_{N-1}\right\}$.

For given integers $m \geq 1$ and $d \geq-1$ define the space of splines

$$
S_{m}^{d}\left(\Delta_{N}\right)=\left\{u \in C^{d}[0, T]:\left.u\right|_{\sigma_{n}} \in \mathcal{P}_{m+d}, n=1, \ldots, N\right\},
$$

where $\mathcal{P}_{k}$ denotes the set of all polynomials with degree not exceeding $k$.

If $d=-1$, then the elements of $S_{m}^{-1}\left(\Delta_{N}\right)$ may have jump discontinuities at the knots $\Delta_{N}$. An element $u \in S_{m}^{d}\left(\Delta_{N}\right)$ as a polynomial spline of degree not greater than $m+d$ can for all $t \in \sigma_{n}, n=1, \ldots, N$, be represented in the form

$$
u_{n}(t)=\sum_{k=0}^{m+d} b_{n k}\left(t-t_{n-1}\right)^{k} .
$$

In order to determine the coefficients $b_{n k}$ we suppose that there exists a fixed selection of collocation parameters $0<c_{1}<\ldots<c_{m} \leq 1$. Then we define collocation points $t_{n j}=t_{n-1}+c_{j} h_{n}, j=1, \ldots, m, n=1, \ldots, N$, forming a set $X(N)$. In order to determine the approximate solution $u \in S_{m}^{d}\left(\Delta_{N}\right)$ of the equation (2.1) we impose the following collocation conditions

$$
u^{\prime}(t)=f(t, u(t))+\int_{0}^{t} \mathcal{K}(t, s, u(s)) d s, \quad t \in X(N) .
$$

Starting the calculations by this method we assume also that we can use the initial values $u_{1}^{(j)}(0)=y^{(j)}(0), j=0, \ldots, d$, which is justified by the requirement $u \in C^{d}[0, T]$. Thus, on every interval $\sigma_{n}$ we have $d+1$ conditions of smoothness and $m$ collocation conditions to determine $m+d+1$ parameters 
$b_{n k}$. This allows us to implement the method step-by-step going from an interval $\sigma_{n}$ to the next one. However, in the case $d=-1$, to be able to use the initial condition on $\sigma_{1}=\left[0, t_{1}\right]$, one collocation condition should be dropped.

\section{THE METHOD IN THE CASE OF A TEST EQUATION}

Consider the test equation

$$
y^{\prime}(t)=\alpha y(t)+\lambda \int_{0}^{t} y(s) d s+f(t), \quad t \in[0, T],
$$

where, in general, $\lambda$ and $\alpha$ may be any complex numbers. Equation (3.1) is considered as the basic test equation (see [1], [2]) in the investigation of stability properties of several methods. Assume that the mesh sequence $\left\{\Delta_{N}\right\}$ is uniform, i.e., $h_{n}=h=T / N$ for all $n$. Representing $t \in \sigma_{n}$ as $t=t_{n-1}+\tau h$, $\tau \in(0,1]$, we have on $\sigma_{n}$

$$
u_{n}\left(t_{n-1}+\tau h\right)=\sum_{k=0}^{m+d} a_{n k} \tau^{k}, \quad \tau \in(0,1]
$$

where we passed to the parameters $a_{n k}=b_{n k} h^{k}$. Using the smoothness conditions (for any $u \in S_{m}^{d}\left(\Delta_{N}\right)$ )

$$
u_{n}^{(j)}\left(t_{n}-0\right)=u_{n+1}^{(j)}\left(t_{n}+0\right), j=0, \ldots, d, n=1, \ldots, N-1,
$$

and collocation conditions (2.3) applied to the test equation (3.1)

$$
\begin{aligned}
u^{\prime}\left(t_{n j}\right)=\alpha u_{n}\left(t_{n j}\right)+\lambda \int_{0}^{t_{n j}} u(s) d s+f\left(t_{n j}\right), j=1, \ldots, & \\
& n=1, \ldots, N,
\end{aligned}
$$

we get the equation in matrix form

$$
\left(V-\alpha h V_{1}-\lambda h^{2} V_{2}\right) \alpha_{n+1}=\left(V_{0}-\alpha h V_{1}-\lambda h^{2}\left(V_{2}-V_{3}\right)\right) \alpha_{n}+h g_{n},
$$

with $(m+d+1) \times(m+d+1)$ matrices $V, V_{0}, V_{1}, V_{2}, V_{3}$ as follows:

$$
V=\left(\begin{array}{c|c}
I \mid 0 \\
\hline C
\end{array}\right), \quad V_{0}=\left(\begin{array}{c|c}
A \mid B \\
\hline C
\end{array}\right)
$$


$I$ being the $(d+1) \times(d+1)$ identity matrix,

$$
C=\left(\begin{array}{ccccc}
0 & 1 & 2 c_{1} & \ldots & (m+d) c_{1}^{m+d-1} \\
\ldots & \ldots & \ldots & \ldots & \ldots \\
0 & 1 & 2 c_{m} & \ldots & (m+d) c_{m}^{m+d-1}
\end{array}\right)
$$

$A$ being a $(d+1) \times(d+1)$ triangular matrix with one's on the main diagonal and zeros below,

$$
\begin{aligned}
& V_{1}=\left(\begin{array}{ccccc} 
& \multicolumn{2}{c}{0} \\
1 & c_{1} & c_{1}^{2} & \ldots & c_{1}^{m+d} \\
\ldots \ldots \ldots \ldots \ldots \ldots \ldots \ldots \\
1 & c_{m} & c_{m}^{2} & \ldots & c_{m}^{m+d}
\end{array}\right) \\
& V_{2}=\left(\begin{array}{cccc} 
& \multicolumn{2}{c}{0} \\
c_{1} & c_{1}^{2} / 2 & \ldots & c_{1}^{m+d+1} /(m+d+1) \\
\ldots \ldots \ldots \ldots \ldots \ldots \ldots \ldots \ldots \ldots \ldots \ldots \\
c_{m} & c_{m}^{2} / 2 & \ldots & c_{m}^{m+d+1} /(m+d+1)
\end{array}\right),
\end{aligned}
$$

$V_{3}$ having first $d+1$ rows 0 and last $m$ rows as $(1,1 / 2, \ldots, 1 /(m+d+1))$, and, finally, the $m+d+1$ dimensional vector $g_{n}=\left(0, \ldots, 0, f\left(t_{n+1,1}\right)-\right.$ $\left.f\left(t_{n 1}\right), \ldots, f\left(t_{n+1, m}\right)-f\left(t_{n m}\right)\right)$. Thus $g_{n}=O(h)$ for $f \in C^{1}$.

In this paper we will focus our main attention on the case $d=-1$, i.e. on piecewise polynomials. Thus, let $d=-1$ in the sequel. Suppose also $\alpha \neq 0$.

Let us introduce the polynomials $P_{k}(\lambda, \alpha)$ and $Q_{k}(\lambda, \alpha)$ by the following recurrence relations

$$
\begin{gathered}
Q_{k}=P_{k-1}+\alpha Q_{k-1}, \\
P_{k}=\lambda Q_{k-1}
\end{gathered}
$$

starting with $P_{0}=0$ and $Q_{0}=1$. Then we have, for example, $P_{1}=\lambda$, $Q_{1}=\alpha, P_{2}=\lambda \alpha, Q_{2}=\lambda+\alpha^{2}$ etc. Combining (3.4) and (3.5) we get also

$$
Q_{k}=\alpha Q_{k-1}+\lambda Q_{k-2} .
$$

Note that for all $k \geq 0$ we have $Q_{k} \neq 0$ or $Q_{k+1} \neq 0$ because the assumption $Q_{k+1}=0$ and $Q_{k}=0$ via (3.6) gives $Q_{k-1}=0, \ldots, Q_{0}=0$, which is not the case. 
Denote by $D_{m}$ Vandermonde's determinant formed by $c_{1}, \ldots, c_{m}$, i.e. $D_{m}=\operatorname{det} V_{1}$ in the case $d=-1$.

Proposition 3.1. We have

$$
\begin{aligned}
\operatorname{det}\left(V-\alpha h V_{1}-\lambda h^{2} V_{2}\right) & =(-1)^{m} Q_{m} D_{m} h^{m} \\
& +(-1)^{m} \lambda Q_{m-1}\left(c_{1}+\cdots+c_{m}\right) D_{m} h^{m+1} / m+O\left(h^{m+2}\right) .
\end{aligned}
$$

Proof. Writing the columns of the determinant as rows with representative element we get

$$
\begin{aligned}
& \operatorname{det}\left(V-\alpha h V_{1}-\lambda h^{2} V_{2}\right)=\left|\begin{array}{c}
-\alpha h-\lambda h^{2} c_{i} \\
1-\alpha h c_{i}-\lambda h^{2} c_{i}^{2} / 2 \\
\ldots \ldots \ldots \ldots \ldots \ldots \ldots \ldots \ldots \ldots \ldots \ldots \ldots \ldots \ldots \ldots \ldots \\
(m-1) c_{i}^{m-2}-\alpha h c_{i}^{m-1}-\lambda h^{2} c_{i}^{m} / m
\end{array}\right| \\
& =-Q_{1} h\left|\begin{array}{c}
1+\left(P_{1} / Q_{1}\right) h c_{i} \\
1-\alpha h c_{i}-\lambda h^{2} c_{i}^{2} / 2 \\
\ldots \ldots \ldots \ldots \ldots \ldots \ldots \ldots \ldots \ldots \ldots \ldots \ldots \ldots \ldots \\
(m-1) c_{i}^{m-2}-\alpha h c_{i}^{m-1}-\lambda h^{2} c_{i}^{m} / m
\end{array}\right|=\ldots \\
& =(-1)^{m} Q_{m} h^{m}\left|\begin{array}{c}
1+\left(P_{1} / Q_{1}\right) h c_{i} \\
c_{i}+\left(P_{2} / Q_{2}\right) h c_{i}^{2} / 2 \\
\ldots \ldots \ldots \ldots \ldots \ldots \ldots \ldots \ldots \\
c_{i}^{m-1}+\left(P_{m} / Q_{m}\right) h c_{i}^{m} / m
\end{array}\right|,
\end{aligned}
$$

which gives the representation (3.7), when $Q_{m} \neq 0, \ldots, Q_{2} \neq 0$. In general case, take the sequences $\lambda_{j} \rightarrow \lambda, \alpha_{j} \rightarrow \alpha$ such that $Q_{k}\left(\lambda_{j}, \alpha_{j}\right) \neq 0$ for all $j$ and $k$. Then from (3.7) for $\lambda_{j}, \alpha_{j}$ we get in limit process (3.7) for $\lambda, \alpha$.

Since $Q_{m} \neq 0$ or $Q_{m-1} \neq 0$, from (3.7) we get

Corollary 3.1. The matrix $V-\alpha h V_{1}-\lambda h^{2} V_{2}$ is invertible for sufficiently small $h$.

\section{STABILITY OF THE METHOD}

Consider the equation (3.3) in the case $d=-1$. Suppose that $\lambda \neq 0$.

DEFINITION 4.1. We say that the spline collocation method by piecewise polynomials is stable if, for any $f \in C^{1}[0, T]$, the approximate solution $u$ of (3.3) remains bounded in $L_{\infty}(0, T)$ as $h \rightarrow 0$. 
Let us notice that the boundedness of $\|u\|_{L_{\infty}(0, T)}$ is equivalent to the boundedness of $\left\|\alpha_{n}\right\|$ in $n$ and $h$ in any fixed norm of $\mathbb{R}^{m}$.

In the case $d=-1$ we have $V_{0}=V$. Then, at least for small $h$, the matrix $W=V-\alpha h V_{1}-\lambda h^{2} V_{2}$ is invertible and (3.3) may be written in form

$$
\alpha_{n+1}=\left(I+\lambda h^{2} W^{-1} V_{3}\right) \alpha_{n}+h W^{-1} g_{n} .
$$

Let us mention for comparison that in the case of Volterra integral equation the same collocation method allows to get the iteration process (see [5])

$$
\alpha_{n+1}=(M+U) \alpha_{n}+r_{n}
$$

with constant matrix $M, U=O(h)$ and $r_{n}=O(h)$ and the stability depends only on the spectral structure of $M$ and is determined completely by collocation parameters $c_{i}$. We will see that the process (4.1) behaves differently.

Proposition 4.1. Matrix $I+\lambda h^{2} W^{-1} V_{3}$ has eigenvalue $\mu=1$ with geometric multiplicity $m-1$.

Proof. It is clear that $\operatorname{Ker}\left(I+\lambda h^{2} W^{-1} V_{3}-\mu I\right)=\operatorname{Ker}\left(W+\lambda h^{2} V_{3}-\mu W\right)$. The geometric multiplicity of $\mu=1$ is $\operatorname{dim} \operatorname{Ker} V_{3}$, but $\operatorname{dim} \operatorname{Ker} V_{3}=m-\operatorname{rank} V_{3}$. As $\operatorname{rank} V_{3}=1$, we get the assertion.

Besides the eigenvalue $\mu=1$ there is one more $\mu \in \operatorname{spec}\left(I+\lambda^{2} W^{-1} V_{3}\right)$ which is equivalent to $\mu-1 \in \operatorname{spec}\left(\lambda h^{2} W^{-1} V_{3}\right)$. Thus, we have to find one additional solution of $\operatorname{det}\left(\lambda h^{2} V_{3}-\mu W\right)=0$ having already 0 as solution of multiplicity $m-1$ by Proposition 4.1.

Denote $A=\lambda h^{2} V_{3}$ and $B=W$ with corresponding entries $a_{i j}$ and $b_{i j}$. Taking into account

$$
a_{11}=\ldots=a_{m 1}, \ldots, a_{1 m}=\ldots=a_{m m}
$$

we get

$$
\operatorname{det}(A-\mu B)=\mu^{m-1}\left|\begin{array}{ccc}
a_{11}-\mu b_{11} & \ldots & a_{1 m}-\mu b_{1 m} \\
b_{11}-b_{21} & \ldots & b_{1 m}-b_{2 m} \\
\ldots \ldots \ldots \ldots \ldots \ldots \ldots \ldots \ldots \ldots \\
b_{11}-b_{m 1} & \ldots & b_{1 m}-b_{m m}
\end{array}\right|
$$

Thus, we have $\operatorname{det}(A-\mu B)=0$ if

$$
\mu=\frac{a_{11} M_{1}+a_{12} M_{2}+\ldots+a_{1 m} M_{m}}{b_{11} M_{1}+b_{12} M_{2}+\ldots+b_{1 m} M_{m}}
$$

with some $M_{i}$ obtained from the determinant in (4.2) 
Proposition 4.2. . We have

$$
M_{1}=Q_{m-1} h^{m-1} D_{m}+P_{m-1} h^{m}\left(c_{1}+\ldots+c_{m}\right) D_{m} / m+O\left(h^{m+1}\right)
$$

with $Q_{m-1} \neq 0$ or $P_{m-1} \neq 0$,

$$
M_{2}=-\lambda Q_{m-2} h^{m} D_{m}-\lambda P_{m-2} h^{m+1}\left(c_{1}+\ldots+c_{m}\right) D_{m} / m+O\left(h^{m+2}\right)
$$

with $Q_{m-2} \neq 0$ or $P_{m-2} \neq 0$,

$$
M_{3}=\lambda^{2} h^{m+1} Q_{m-3} D_{m} / 2+O\left(h^{m+2}\right)
$$

and

$$
M_{k}=O\left(h^{m+2}\right), k \geq 4 .
$$

Proof. Writing again the columns of the determinant as rows we get

$$
\begin{aligned}
& M_{1}=\left|\begin{array}{c}
\alpha h\left(c_{i}-c_{1}\right)+\lambda h^{2}\left(c_{i}^{2}-c_{1}^{2}\right) / 2 \\
2\left(c_{1}-c_{i}\right)+\alpha h\left(c_{i}^{2}-c_{1}^{2}\right)+\lambda h^{2}\left(c_{i}^{3}-c_{1}^{3}\right) / 3 \\
\ldots \ldots \ldots \ldots \ldots \ldots \ldots \ldots \ldots \ldots \ldots \ldots \ldots \ldots \ldots \ldots \ldots \ldots \ldots \ldots \ldots \ldots \ldots \ldots \ldots \ldots \ldots \ldots \ldots \ldots \ldots \\
(m-1)\left(c_{1}^{m-2}-c_{i}^{m-2}\right)+\alpha h\left(c_{i}^{m-1}-c_{1}^{m-1}\right)+\lambda h^{2}\left(c_{i}^{m}-c_{1}^{m}\right) / m
\end{array}\right| \\
& =Q_{1} h\left|\begin{array}{c}
c_{i}-c_{1}+\left(P_{1} / Q_{1}\right) h\left(c_{i}^{2}-c_{1}^{2}\right) / 2 \\
\left(Q_{2} / P_{1}\right) h\left(c_{i}^{2}-c_{1}^{2}\right)+\lambda h^{2}\left(c_{i}^{3}-c_{1}^{3}\right) / 3 \\
\ldots \ldots \ldots \ldots \ldots \ldots \ldots \ldots \ldots \ldots \ldots \ldots \ldots \ldots \ldots \ldots \ldots \ldots \ldots \ldots \ldots \ldots \ldots \ldots \ldots \ldots \ldots \ldots \ldots \ldots \ldots \ldots \ldots \\
(m-1)\left(c_{1}^{m-2}-c_{i}^{m-2}\right)+\alpha h\left(c_{i}^{m-1}-c_{1}^{m-1}\right)+\lambda h^{2}\left(c_{i}^{m}-c_{1}^{m}\right) / m
\end{array}\right|
\end{aligned}
$$

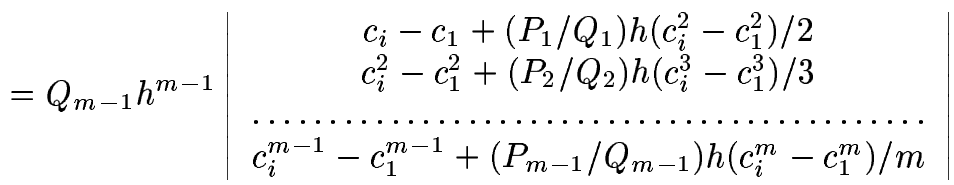

which gives (4.4) after some straightforwards calculations with determinants. As in the proof of Proposition 3.1, this argument is correct if $Q_{m-1} \neq 0$, $\ldots, Q_{1} \neq 0$, but in general case the limit process will arrange the proof. The other formulae (4.5)-(4.7) can be obtained by similar calculations.

Proposition 4.3. For the solution (4.3) it holds

1) if $Q_{m-1} \neq 0$ and $Q_{m} \neq 0$ then $\mu=-\lambda\left(Q_{m-1} / Q_{m}\right) h+O\left(h^{2}\right)$,

2) if $Q_{m-1} \neq 0$ and $Q_{m}=0$ then $\mu=-m /\left(c_{1}+\ldots+c_{m}\right)+O(h)$,

3) if $Q_{m-1}=0$ and $Q_{m} \neq 0$ then $\mu=O\left(h^{2}\right)$. 
Proof. The main term in the numerator of (4.3) is $\lambda h^{m+1} Q_{m-1} D_{m}$ for $Q_{m-1} \neq 0$ and $O\left(h^{m+2}\right)$ for $Q_{m-1}=0$. In the denominator of (4.3) the coefficient of $h^{m}$ is $-Q_{m} D_{m}$, thus the first and third assertion follow immediately. The coefficient of $h^{m+1}$ can be found as $-\lambda\left(c_{1}+\ldots+c_{m}\right) D_{m} Q_{m-1} / m$ which yields the formula for $\mu$ in second case.

Remark 4.1. It is natural to ask whether $\mu$ in (4.3) may have higher order in $h$ than 2 ? In fact, more detailed calculations show that

$$
\begin{gathered}
M_{1}=Q_{m-1} h^{m-1} D_{m}+\lambda Q_{m-2} h^{m} \text { sym }_{1} D_{m} / m \\
+\lambda^{2} Q_{m-3} h^{m+1} \operatorname{sym}_{2} D_{m} / m(m-1)+\ldots \\
+\lambda^{m-1} Q_{0} h^{2 m-2} \text { sym }_{m-1} D_{m} / m ! \\
M_{2}=-\lambda Q_{m-2} h^{m} D_{m}-\lambda^{2} Q_{m-3} h^{m-3} \operatorname{sym}_{1} D_{m} / m-\ldots \\
-2 \lambda^{m-1} Q_{0} h^{2 m-2} \operatorname{sym}_{m-2} D_{m} / m !
\end{gathered}
$$

where $\operatorname{sym}_{i}$ are standard symmetrical polynomials of $c_{1}, \ldots, c_{m}$ of order $i$, for example, sym ${ }_{1}=c_{1}+\ldots+c_{m}, s m_{2}=c_{1} c_{2}+\ldots+c_{m-1} c_{m}$. Basing on these developments it is possible to show with quite complicated calculations that $\mu=\nu h^{k}+O\left(h^{k+1}\right), \nu \neq 0$, for $k>3$ is not possible, but in the case $Q_{m-1}=0, Q_{m} \neq 0$ for $c_{1}+\ldots+c_{m} \neq m / 2$ we have $\mu=\nu h^{2}+O\left(h^{3}\right), \nu \neq 0$, and for $c_{1}+\ldots+c_{m}=m / 2$ we get $\mu=\nu h^{3}+O\left(h^{4}\right), \nu \neq 0$.

Thus, the matrix $I+\lambda h^{2} W^{-1} V_{3}$, which we denote here by $M$, has eigenvalues with equal algebraic and geometric multiplicities. This implies that its Jordan form is diagonal matrix with $m-1$ entries 1 and one $1+\epsilon$ with $\epsilon=O\left(h^{k}\right), k=0, \ldots, 3$. The Jordan representation $M=P J P^{-1}$ gives $M^{n}=P J^{n} P^{-1}$ and at least for $k \geq 1$ the matrix $J^{n}$ is bounded. We see that the boundedness of $M^{n}$ depends also on behavior of $P$ and $P^{-1}$ in process $h \rightarrow 0$. It can be directly checked that the matrix $\lambda h^{2} W^{-1} V_{3}$ or $W^{-1} V_{3}$ has the same eigenvectors as $M$ and they are the columns of $P$. Take them as an orthonormal system $p^{1}, \ldots, p^{m-1}$ corresponding to $0 \in \operatorname{spec}\left(W^{-1} V_{3}\right)$, which give $p^{1}, \ldots, p^{m-1} \in \operatorname{Ker} V_{3}$, and $p^{m}$ of Euclidean norm 1 corresponding to $\epsilon \in \operatorname{spec}\left(W^{-1} V_{3}\right)$. Clearly $P$ is bounded. The boundedness of $P^{-1}$ can be guaranteed if $|\operatorname{det} P| \geq \delta$ for some $\delta>0$. This takes place if we get $<p, q>\leq \sigma\|p\|\|q\|$ with $\sigma<1$ for all $p \in \operatorname{Ker} V_{3}$ and all $q \in \operatorname{Ker}\left(\lambda h^{2} V_{3}-\mu W\right)$ which is equivalent to $\langle p, q\rangle \leq \sigma\|p\|\|q\|$ for all $p \in\left(\operatorname{Ker} V_{3}\right)^{\perp}$ and all $q \in\left(\operatorname{Ker}\left(\lambda h^{2} V_{3}-\mu W\right)^{\perp}\right.$. Here we may consider $p=(1,1 / 2, \ldots, 1 / m)$ because $\operatorname{dim}\left(\operatorname{Ker} V_{3}\right)^{\perp}=1$ and $q=\sum_{1 \leq j \leq m-1} \lambda_{j} q^{j}$ with $q^{j}$ (we write $q^{j}$ here 
in column)

$$
q^{j}=\left(\begin{array}{l}
\lambda h^{2}-\mu\left(\left(-\alpha h-\lambda h^{2} c_{j}\right)\right. \\
\lambda h^{2} / 2-\mu\left(1-\alpha h c_{j}-\lambda h^{2} c_{j}^{2} / 2\right) \\
\cdots \\
\lambda h^{2} / m-\mu\left((m-1) c_{j}^{m-2}-\alpha h c_{j}^{m-1}-\lambda h^{2} c_{j}^{m} / m\right.
\end{array}\right)
$$

as $q^{1}, \ldots, q^{m-1}$ give a basis in $\left(\operatorname{Ker}\left(h^{2} V_{3}-\mu W\right)\right)^{\perp}$ at least for small $h$. Let $\bar{q}^{j}=q^{j}-\lambda h^{2} p$. Since $\operatorname{det} W \neq 0, \bar{q}^{1}, \ldots, \bar{q}^{m-1}$ are linearly independent. Similarly, we get also the linear independence of $p, \bar{q}^{1}, \ldots, \bar{q}^{m-1}$ for small $h$. Then

$$
\langle p, q\rangle=\left(\sum_{j=1}^{m-1} \lambda_{j}\right) \lambda h^{2}\left\langle p, p>-\mu\left\langle p, \sum_{j=1}^{m-1} \lambda_{j} \bar{q}^{j}\right\rangle .\right.
$$

We may consider only the "worse" case, namely, when $q$ is the projection of $p$ onto $\left(\operatorname{Ker}\left(\lambda h^{2} V_{3}-\mu W\right)\right)^{\perp}$. Then in the process $h \rightarrow 0$ the coefficients $\lambda_{j}$ stabilize and

$$
\left\langle p, \sum_{j=1}^{m-1} \lambda_{j} \bar{q}^{j}\right\rangle \approx \sigma_{0}\|p\|\left\|\sum_{j=1}^{m-1} \lambda_{j} \bar{q}^{j}\right\|
$$

for some fixed $\sigma_{0} \in(-1,1)$ due to the linear independence of $p, \bar{q}^{1}, \ldots, \bar{q}^{m}$. In the cases $\mu \sim \nu h, \nu \neq 0$, and $\mu \sim$ const the last term in (4.8) is dominant and we get $\left\langle p, q>\leq \sigma\|p\|\|q\|\right.$ with $\sigma<1$ (actually, $\sigma \rightarrow \sigma_{0}$ ).

Note that the case $\mu \sim \nu h^{k}, \nu \neq 0, k \geq 2$ needs additional analysis but similar arguments lead us also to the boundedness of $M^{n}$.

Summing up the results of presented reasonings and Proposition 4.3 we have

\section{Proposition 4.4. The following holds}

1) if $Q_{m} \neq 0$ then the method is stable,

2) if $Q_{m}=0$ (and hence $Q_{m-1} \neq 0$ ) then for $c_{1}+\ldots+c_{m} \geq m / 2$ the method is stable, for $c_{1}+\ldots+c_{m}<m / 2$ unstable.

For example, let $m=3$. We have $Q_{3}=2 \lambda \alpha+\alpha^{3}$. For $2 \lambda \alpha+\alpha^{3} \neq 0$ the method is stable and for $2 \lambda \alpha+\alpha^{3}=0$ the stability region is $c_{1}+c_{2}+c_{3} \geq 3 / 2$.

\section{NUMERICAL TESTS}

We use the test equation (3.1) with the solution $y(t)=\left(\sin t+\cos t+e^{t}\right) / 2$. As an approximate value of $\|u\|_{\infty}$ we calculated $\max _{1 \leq n \leq N} \max _{0 \leq k \leq 10}\left|u_{n}\left(t_{n-1}+k h / 10\right)\right|$. The results are presented in following tables. We can see different dependence 
of the stability on the cases $Q_{m}=0$ and $Q_{m} \neq 0$, as well as on different choices of $c_{i}$, quantitatively in accordance to the theoretical results.

Case $m=2$

\begin{tabular}{cccccc}
$\alpha=1, \lambda=1$ & \multicolumn{5}{c}{} \\
\hline $\mathrm{N}$ & 4 & 16 & 64 & 256 & 1024 \\
\hline $\begin{array}{l}c_{1}=0.1 \\
c_{2}=0.2\end{array}$ & 1.81554 & 1.77374 & 1.76551 & 1.76302 & 1.76254 \\
\hline $\begin{array}{l}c_{1}=0.5 \\
c_{2}=1.0\end{array}$ & 1.78039 & 1.76705 & 1.76353 & 1.76264 & 1.76242 \\
\hline
\end{tabular}

\begin{tabular}{cccccc}
\multicolumn{6}{l}{$\alpha=1, \lambda=-1$} \\
\hline $\mathrm{N}$ & 4 & 16 & 64 & 256 & 1024 \\
\hline$c_{1}=0.2$ & 5.01 & 12.76 & $1.27 \cdot 10^{15}$ & $1.32 \cdot 10^{66}$ & $9.82 \cdot 10^{271}$ \\
$c_{2}=0.5$ & & & & & \\
\hline$c_{1}=0.3$ & 3.71489 & 3.59568 & 3.56853 & 3.56193 & 3.56029 \\
$c_{2}=0.7$ & & & & & \\
\hline$c_{1}=0.5$ & 3.48369 & 3.52181 & 3.55030 & 3.55739 & 3.55916 \\
$c_{2}=1.0$ & & & & & \\
\hline
\end{tabular}

Case $m=3$

\begin{tabular}{|c|c|c|c|c|c|}
\hline $\mathrm{N}$ & 4 & 16 & 64 & 256 & 1024 \\
\hline $\begin{array}{l}c_{1}=0.1 \\
c_{2}=0.2 \\
c_{3}=0.3\end{array}$ & 1.64625 & 1.66144 & 1.66684 & 1.66827 & 1.66863 \\
\hline $\begin{array}{l}c_{1}=0.2 \\
c_{2}=0.5 \\
c_{3}=0.9\end{array}$ & 1.65331 & 1.66493 & 1.66779 & 1.66851 & 1.66869 \\
\hline$\alpha=2, \lambda$ & -2 & & & & \\
\hline $\mathrm{N}$ & 4 & 16 & 64 & & $\overline{56}$ \\
\hline $\begin{array}{l}c_{1}=0.1 \\
c_{2}=0.2 \\
c_{3}=0.3\end{array}$ & 58.07 & $9.08 \cdot 10^{8}$ & $7.11 \cdot 10^{37}$ & 2.79 & $10^{153}$ \\
\hline $\begin{array}{l}c_{1}=0.2 \\
c_{2}=0.5 \\
c_{3}=0.8\end{array}$ & 3.82646 & 3.78772 & 3.77323 & & 6931 \\
\hline $\begin{array}{l}c_{1}=0.3 \\
c_{2}=0.6 \\
c_{3}=0.9\end{array}$ & 3.02214 & 2.56511 & 2.56229 & & 6247 \\
\hline
\end{tabular}




\section{REFERENCES}

[1] H. Brunner and P. J. van der Houwen. The numerical solution of Volterra equations. North-Holland, Amsterdam, 1986.

[2] H.Brunner and J. D. Lambert. Stability of numerical methods for Volterra integrodifferential equation. Computing, 12 1974, 75 - 89.

[3] I. Danciu. Numerical stability of collocation methods for Volterra integro-differential equations. Rev. Anal. Numér. Théorie Approximation, 26 (1-2), 1997, 59-74.

[4] H. -S. Hung. The numerical solution of differential and integral equations by spline functions. MRC Tech. report, 1053 University of Wisconsin, Madison.1970

[5] P. Oja. Stability of the spline collocation method for Volterra integral equations. $J$. Integral Equations Appl., 13 (2), 2001.

[6] P.Oja. Stability of collocation by smooth splines for Volterra integral equations. In: Proc. of the Intern. Conference, Oslo, 2000, Mathemathical methods for curves and surfaces, $2001,405-412$.

\section{Kolokaciju metodo stabilumas Voltero tipo integro-diferencialinėms lygtims}

P. Oja, M. Tarang

Voltero tipo integro-diferencialinè lygtis sprendžiama kolokaciju metodu. Ištirtas metodo stabilumas, kai naudojami trūkūs splainai. Parodyta, kad stabilumas priklauso nuo kolokaciniu parametrų ir nuo vienos testinès lygties parametrų. Pateikiami skaičiavimo eksperimento rezultatai, kurie patvirtina visus teorinius teiginius. 